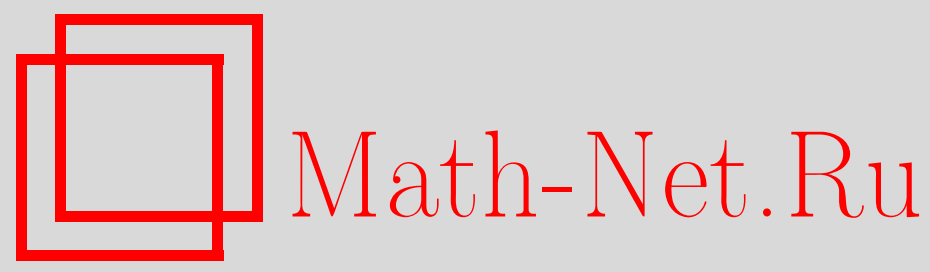

В. Н. Голощапов, П. В. Ролдугин, Оценка числа переменных булевых функций небольшого веса, не содержащих имплицент меньшей длины, Дискрет. матем., 2013, том 25, выпуск 1, 45-62

DOI: https://doi.org/10.4213/dm1220

Использование Общероссийского математического портала Math-Net.Ru подразумевает, что вы прочитали и согласны с пользовательским соглашением http://www . mathnet.ru/rus/agreement

Параметры загрузки:

IP : 54.162 .85 .209

26 апреля 2023 г., 05:22:18 


\section{Оценка числа переменных булевых функций небольшого веса, не содержащих имплицент меньшей длины}

() 2013 г. В. Н. Голощапов, П. В. Ролдугин

В статье рассматривается величина $n_{\max }^{(k)}(m)$ - максимальное количество переменных булевых функций фиксированного веса $m$, не имеющих имплицент от $k$ неизвестных. Полученные результаты о строении множества выполняющих векторов таких функций позволили получить оценки и, в ряде случаев, точные значения величины $n_{\max }^{(k)}(m)$ для $m<3 \cdot 2^{k-1}, k \geqslant 3$.

\section{1. Введение}

Будем использовать следующие обозначения и определения. Через $V_{n}$ обозначим множество двоичных векторов длины $n$. Для булевой функции $f$ от $n$ переменных обозначим через $E_{f}$ множество ее выполняющих векторов, то есть

$$
E_{f}=\left\{\left(\alpha_{1}, \ldots, \alpha_{n}\right) \in V_{n}: f\left(\alpha_{1}, \ldots, \alpha_{n}\right)=1\right\} ;
$$

весом функции $f$ назовем величину

$$
\|f\|=\left|E_{f}\right| .
$$

Имплицентой булевой функции $f$ является не равная константе булева функция $g$, такая что $f \cdot g=f$, что эквивалентно условию $E_{f} \subseteq E_{g}$, см. [1]. Иногда (см. [3]) используют понятие аннулятора булевой функции $f$ : аннулятором называется такая функция $h$, что $f \cdot h=0$. Очевидно, что $h-$ аннулятор функции $f$ тогда и только тогда, когда $\bar{h}=h \oplus 1$ является имплицентой функции $f$. В задаче минимизации ДНФ часто встречается понятие импликанты булевой функции $f$ (см. [4]): импликантой называется такая функция $q$, что $f \cdot q=q$. Также очевидно, что $q-$ импликанта функции $f$ тогда и только тогда, когда $\bar{q}$ является имплицентой функции $\bar{f}$.

Длиной имплиценты назовем число ее существенных переменных; длину функции, равной фиксированной константе (0 или 1$)$ на любых аргументах, будем считать равной 0 . Если функция $f\left(x_{1}, \ldots, x_{n}\right)$ отлична от константы, то обязательно существует имплицента функции $f$ длины $n$ - сама функция $f$. Функцией, не имеющей имплицент, отличных от $f$, является булева функция

$$
f\left(x_{1}, \ldots, x_{n}\right)=x_{1}^{\alpha_{1}} \vee x_{2}^{\alpha_{2}} \vee \ldots \vee x_{n}^{\alpha_{n}}
$$


для некоторого произвольного вектора $\left(\alpha_{1}, \ldots, \alpha_{n}\right) \in V_{n}$. Поскольку для такой функции $\|f\|=2^{n}-1$, условие $E_{f} \subseteq E_{g}$ может выполняться только для функций $g \equiv 1$ или $g=f$. Для функций, имеющих вес меньше, чем $2^{n}-1$, обязательно существует имплицента, отличная от $f$. При этом число существенных переменных имплиценты может совпадать или быть меньше, чем $n$. Приведем пример функции, у которой есть имплиценты, не совпадающие с $f$, но нет имплицент с числом существенных переменных, меньшим $n$.

Пример 1. Рассмотрим функцию

$$
f\left(x_{1}, \ldots, x_{n}\right)=x_{1} \oplus x_{2} \oplus \ldots \oplus x_{n} .
$$

Предположим, что функция $g$ от $k$ переменных является имплицентой функции $f$, и $1 \leqslant k<n$. Без ограничения общности считаем, что $g$ зависит от переменных $x_{1}, \ldots, x_{k}$. По определению, $E_{f} \subset E_{g}$, то есть $f \cdot g=f$. Следовательно, справедлива следующая цепочка равенств:

$$
\begin{aligned}
f\left(x_{1}, \ldots, x_{n}\right) g\left(x_{1}, \ldots, x_{k}\right)= & \left(x_{1} \oplus \ldots \oplus x_{n}\right) g\left(x_{1}, \ldots, x_{k}\right) \\
= & \left(x_{1} \oplus \ldots \oplus x_{k}\right) g\left(x_{1}, \ldots, x_{k}\right) \\
& \oplus\left(x_{k+1} \oplus \ldots \oplus x_{n}\right) g\left(x_{1}, \ldots, x_{k}\right) \\
= & f\left(x_{1}, \ldots, x_{n}\right)=x_{1} \oplus \ldots \oplus x_{n} .
\end{aligned}
$$

Отсюда получаем, что

$$
\left(x_{1} \oplus \ldots \oplus x_{k}\right) \bar{g}\left(x_{1}, \ldots, x_{k}\right)=\left(x_{k+1} \oplus \ldots \oplus x_{n}\right) \bar{g}\left(x_{1}, \ldots, x_{k}\right) .
$$

По определению имплиценты, $g$ отлична от константы, следовательно, существует вектор $\left(\alpha_{1}, \ldots, \alpha_{k}\right)$, такой, что $g\left(\alpha_{1}, \ldots, \alpha_{k}\right)=0$. Выберем $\left(\alpha_{k+1}, \ldots, \alpha_{n}\right) \in V_{n-k}$ следующим образом: $\alpha_{k+1}, \ldots, \alpha_{n-1}$ определим произвольно, и положим

$$
\alpha_{n}=\alpha_{1} \oplus \ldots \oplus \alpha_{k} \oplus \alpha_{k+1} \oplus \ldots \oplus \alpha_{n-1} \oplus 1 .
$$

Подставляя вместо переменных $x_{1}, \ldots, x_{n}$ значения $\alpha_{1}, \ldots, \alpha_{n}$, получаем из (1) равенство

$$
\alpha_{1} \oplus \ldots \oplus \alpha_{k}=\alpha_{k+1} \oplus \ldots \oplus \alpha_{n}
$$

что противоречит выбору $\alpha_{n}$. Следовательно, у функции $f$ нет имплицент длины $k$.

Для фиксированных натуральных значений $n$ и $m$ обозначим через $F_{n, m}$ множество всех булевых функций от $n$ переменных веса $m$. Ясно, что множество $F_{n, m}$ не пусто тогда и только тогда, когда $m \leqslant 2^{n}$. Далее для натуральных значений $m, n, k, n>k$, рассмотрим множество

$$
N_{n, m, k}=\left\{f \in F_{n, m}: f \text { не имеет имплицент длины } k\right\} .
$$

Если при фиксированных значениях $m$ и $k$ существует хотя бы одно непустое множество $N_{n, m, k}$, то рассмотрим величину

$$
n_{\max }^{(k)}(m)=\max \left\{n: N_{n, m, k} \neq \varnothing\right\} .
$$

Другими словами, $n_{\max }^{(k)}(m)$ равно максимальному числу переменных у булевой функции веса $m$, не имеющей имплицент длины $k$. Отметим, что в случае, когда существует бесконечно много значений $n$, для которых множество $N_{n, m, k}$ непусто, при фиксированных 
значениях $m$ и $k$, возможно равенство $n_{\max }^{(k)}(m)=\infty$. Далее мы покажем, что $n_{\max }^{(k)}(m)=\infty$ только при $k=1$ и $m \geqslant 2$. Если при всех значениях $n$ множества $N_{n, m, k}$ пусты, тогда положим $n_{\max }^{(k)}(m)=0$.

Рассмотрим ряд элементарных свойств функции $n_{\max }^{(k)}(m)$.

Лемма 1. Пусть $m$ и $k$ - натуральнье числа. Тогда

(1) $n_{\max }^{(1)}(1)=0$;

(2) если $m \geqslant 2$, то $n_{\max }^{(1)}(m)=\infty$;

(3) $n_{\max }^{(k)}(m) \geqslant n_{\max }^{(k+1)}(m)$;

(4) если $k \geqslant 2$, то $n_{\max }^{(k)}(m) \leqslant 2^{m}$;

(5) если $k \geqslant 2$ и $1 \leqslant m<2^{k}$, то $n_{\max }^{(k)}(m)=0$;

(6) если $k \geqslant 2 u m \geqslant 2^{k}$, то $n_{\max }^{(k)}(m)>\log _{2} m$;

(7) если $k \geqslant 2$ и $m \geqslant 2^{k}$, то $n_{\max }^{(k)}(m) \geqslant k+1$;

(8) если $k \geqslant 2$, то $n_{\max }^{(k)}(m+1) \geqslant n_{\max }^{(k)}(m)$.

Доказательство. Пусть $n>1$. Если булева функция $f$ от $n$ переменных имеет вес 1 , то

$$
E_{f}=\left\{\left(\alpha_{1}, \ldots, \alpha_{n}\right)\right\},
$$

поэтому

$$
f\left(x_{1}, \ldots, x_{n}\right)=x_{1}^{\alpha_{1}} \cdots x_{n}^{\alpha_{n}}
$$

Любая функция

$$
g=x_{i}^{\alpha_{i}}, \quad i=1, \ldots, n,
$$

является имплицентой $f$ длины 1 , поэтому все множества $N_{n, 1,1}$ пусты и

$$
n_{\max }^{(1)}(1)=0 .
$$

Пункт 1 доказан.

Пусть теперь $m \geqslant 2$. Для любого $n>1$, такого что $m<2^{n}$, рассмотрим любую такую функцию $f \in F_{n, m}$, что $\{(0, \ldots, 0),(1, \ldots, 1)\} \subseteq E_{f}$. Поскольку $m \neq 2^{n}$, функция $f$ не равна константе. Далее,

$$
f\left(x_{1}, \ldots, x_{n}\right)=x_{1} \cdots x_{n} \vee \bar{x}_{1} \cdots \bar{x}_{n} \vee f^{\prime}\left(x_{1}, \ldots, x_{n}\right),
$$

где $f^{\prime}-$ булева функция от переменных $x_{1}, \ldots, x_{n}$, такая, что

$$
E_{f^{\prime}}=E_{f} \backslash\{(0, \ldots, 0),(1, \ldots, 1)\} .
$$

Покажем, что $f$ не имеет имплицент длины 1 . Действительно, для любого $i=1, \ldots, n$ и $\alpha \in\{0,1\}$ верно, что

$$
x_{i}^{\alpha} f=x_{1}^{\alpha} \cdots x_{n}^{\alpha} \vee f^{\prime} \neq x_{1} \cdots x_{n} \vee \bar{x}_{1} \cdots \bar{x}_{n} \vee f^{\prime}=f ;
$$


поскольку $f^{\prime}\left(\bar{\alpha}_{1}, \ldots, \bar{\alpha}_{n}\right)=0$, левая часть на наборе $\left(\bar{\alpha}_{1}, \ldots, \bar{\alpha}_{n}\right)$ равна 0, а правая часть равна 1. Таким образом, для любого такого $n$, что $m<2^{n}$, справедливо $N_{n, m, k} \neq \varnothing$ и, следовательно,

$$
n_{\max }^{(1)}(m)=\infty
$$

Пункт 2 доказан.

Пусть $n>k+1$. Предположим, что $N_{n, m, k}=\varnothing$. Тогда либо пусто множество $F_{n, m}$ и, следовательно, $N_{n, m, k+1}=\varnothing$; либо любая функция из $F_{n, m}$ имеет имплиценту длины $k$. Если $g-$ имплицента длины $k$ функции $f\left(x_{1}, \ldots, x_{n}\right) \in F_{n, m}$, и $x_{i}$ - несущественная переменная функции $g$, то функция $x_{i} \vee g$ также имплицента функции $f$, причем длины $k+1$. Следовательно, любая функция из $F_{n, m}$ имеет имплиценту длины $k+1$, поэтому $N_{n, m, k+1}=\varnothing$. Иными словами, при $n>k+1$ из равенства $N_{n, m, k}=\varnothing$ следует тождество $N_{n, m, k+1}=\varnothing$, и это доказывает неравенство

$$
n_{\max }^{(k)}(m) \geqslant n_{\max }^{(k+1)}(m) .
$$

Пункт 3 доказан.

Пусть $m, n \in \mathbf{N}$. Докажем сначала, что $n_{\max }^{(2)}(m) \leqslant 2^{m}$. Покажем, что если $n>2^{m}$, то у любой функции $f$ от $n$ переменных веса $m$ найдется имплицента длины 2 . Это будет означать, что при $n>2^{m}$ все множества $N_{n, m, 2}$ пусты и, следовательно, $n_{\max }^{(2)}(m) \leqslant 2^{m}$. Рассмотрим произвольную функцию $f\left(x_{1}, \ldots, x_{n}\right)$ веса $m$. Пусть множество ее выполняющих векторов есть

$$
E_{f}=\left\{\left(\alpha_{1}^{(i)}, \ldots, \alpha_{n}^{(i)}\right): i=1, \ldots, m\right\}
$$

Рассмотрим для каждого $j=1, \ldots, n$ битовые векторы $\left(\alpha_{j}^{(1)}, \alpha_{j}^{(2)}, \ldots, \alpha_{j}^{(m)}\right)$. Это векторы длины $m$, и их $n$ штук. Поскольку $n>2^{m}$, среди указанных векторов найдутся два одинаковых вектора $\left(\alpha_{u}^{(1)}, \alpha_{u}^{(2)}, \ldots, \alpha_{u}^{(m)}\right)=\left(\alpha_{v}^{(1)}, \alpha_{v}^{(2)}, \ldots, \alpha_{v}^{(m)}\right), u, v=1, \ldots, n, u \neq v$. Это означает, что если $f\left(x_{1}, \ldots, x_{n}\right)=1$, то $x_{u}=x_{v}$. Тогда функция

$$
g=x_{u} \vee \bar{x}_{v}
$$

является имплицентой функции $f$. Действительно, если $f\left(x_{1}, \ldots, x_{n}\right)=1$, то $x_{u}=x_{v}$ и, следовательно,

$$
g=x_{u} \vee \bar{x}_{v}=x_{u} \vee \bar{x}_{u}=1
$$

Иными словами, $E_{f} \subseteq E_{g}$, поэтому

$$
n_{\max }^{(2)}(m) \leqslant 2^{m}
$$

Осталось заметить, что по п. 3 при $k \geqslant 2$ выполняется соотношение

$$
n_{\max }^{(k)}(m) \leqslant n_{\max }^{(2)}(m) \leqslant 2^{m} .
$$

Пункт 4 доказан.

Рассмотрим $n>k$. Для тех $n$, при которых $m>2^{n}$, верно, что $F_{n, m}=\varnothing$ и, поскольку $N_{n, m, k} \subset F_{n, m}$, верно, что $N_{n, m, k}=\varnothing$. Зафиксируем произвольное значение $n$, при котором $F_{n, m} \neq \varnothing$, то есть $m \leqslant 2^{n}$. Пусть $f \in F_{n, m}$. Рассмотрим множество $k$-мерных двоичных векторов $\left\{\left(\alpha_{1}, \ldots, \alpha_{k}\right)\right.$ : существуют такие $\alpha_{k+1}, \ldots, \alpha_{n}$, что $\left.f\left(\alpha_{1}, \ldots, \alpha_{n}\right)=1\right\}$. Так 
как вес функции $f$ равен $m$ и по условию $m<2^{k}$, то это множество не может содержать все векторы длины $k$; обозначим отсутствующий вектор через $\left(\alpha_{1}^{\prime}, \ldots, \alpha_{k}^{\prime}\right)$. Далее, возьмем функцию

$$
g\left(x_{1}, \ldots, x_{n}\right)=x_{1}^{\alpha_{1}^{\prime}} \ldots x_{k}^{\alpha_{k}^{\prime}} \oplus 1 .
$$

Длина функции $g$ равна $k$. Если на некотором векторе $\left(x_{1}, \ldots, x_{n}\right)$ выполнено равенство $f\left(x_{1}, \ldots, x_{n}\right)=1$, то $\left(x_{1}, \ldots, x_{k}\right) \neq\left(\alpha_{1}, \ldots, \alpha_{k}\right)$, и поэтому $g\left(x_{1}, \ldots, x_{n}\right)=1$. Иными словами, $E_{f} \subset E_{g}$, что означает, что $g$ является имплицентой длины $k$ функции $f$. Таким образом, для каждой функции $f \in F_{n, m}$ существует имплицента длины $k$, то есть $N_{n, m, k}=\varnothing$. Значит, при всех значениях $n N_{n, m, k}=\varnothing$, и получаем, что

$$
n_{\max }^{(k)}(m)=0 .
$$

Пункт 5 доказан.

Если существует хотя бы одно значение $n, n>\log _{2} m$, при котором $N_{n, m, k} \neq \varnothing$, то

$$
n_{\max }^{(k)}(m)>\log _{2} m .
$$

Иными словами, достаточно привести хотя бы одну функцию $f \in F_{n, m}, n>\log _{2} m$, не имеющую имплицент длины $k$. Возьмем

$$
n_{0}=\left[\log _{2} m\right]+1,
$$

тогда $n_{0}>\log _{2} m$. Докажем неравенство

$$
0 \leqslant m-2^{n_{0}-1}<2^{n_{0}-1} .
$$

Действительно, $n_{0}>\log _{2} m$, следовательно, $m<2^{n_{0}}$ или $m-2^{n_{0}-1}<2^{n_{0}-1}$. С другой стороны,

$$
\log _{2} m \geqslant\left[\log _{2} m\right]=n_{0}-1,
$$

поэтому $m \geqslant 2^{n_{0}-1}$ или $0 \leqslant m-2^{n_{0}-1}$. Далее, рассмотрим функцию

$$
f^{\prime}\left(x_{1}, \ldots, x_{n_{0}}\right)=x_{1} \oplus x_{2} \oplus \ldots \oplus x_{n_{0}} .
$$

Тогда

$$
\left\|f^{\prime}\right\|=\left|E_{f^{\prime}}\right|=2^{n_{0}-1}
$$

Добавим к множеству $E_{f^{\prime}}$ еще $m-2^{n_{0}-1}$ новых различных $n_{0}$-мерных двоичных векторов, выбрав их произвольным образом; это возможно сделать именно ввиду неравенства $0 \leqslant m-2^{n_{0}-1}<2^{n_{0}-1}$. Полученное множество является множеством выполняющих векторов некоторой функции $f$, причем $f \in F_{n_{0}, m}$. Предположим, что функция $g$ от $k$ переменных является имплицентой функции $f$. По определению, $E_{f} \subset E_{g}$; так как, по построению $f$, справедливо включение $E_{f^{\prime}} \subset E_{f}$, верно, что $E_{f^{\prime}} \subset E_{g}$, иными словами, $g$ есть имплицента функции $f^{\prime}$. Но, как показано в примере выше, функция $f^{\prime}$ не имеет имплицент от числа переменных, меньшего $n_{0}$. По условию, $m \geqslant 2^{k}$, из этого следует, что $k \leqslant \log _{2} m<n_{0}$, и получаем, что у функции $f$ нет имплицент длины $k$. Таким образом, $N_{n_{0}, m, k} \neq \varnothing$ и $n_{0}>\log _{2} m$, то есть

$$
n_{\max }^{(k)}(m)>\log _{2} m .
$$


Пункт 6 доказан.

Согласно п. 6 ,

$$
n_{\max }^{(k)}(m)>\log _{2} m,
$$

а так как, по условию, $m \geqslant 2^{k}$, верно, что

$$
n_{\text {max }}^{(k)}(m)>\log _{2} m \geqslant \log _{2} 2^{k}=k .
$$

Следовательно,

$$
n_{\max }^{(k)}(m)>k
$$

Пункт 7 доказан.

По п. 5 ,

$$
n_{\max }^{(k)}(2)=n_{\max }^{(k)}(3)=\ldots=n_{\max }^{(k)}\left(2^{k}-1\right)=0 .
$$

Пусть $m \geqslant 2^{k}$. Обозначим

$$
n_{0}=n_{\max }^{(k)}(m) .
$$

Из п. 4 и 6 следует, что $0<n_{0}<\infty$. Это означает, что существует функция $f \in F_{n_{0}, m}$, которая не имеет имплицент длины $k$. Из п. 6 также следует, что $n_{0}>\log _{2} m$, то есть $m<2^{n_{0}}$, поэтому множество $E_{f}$ не содержит всех векторов длины $n_{0}$. Добавим в $E_{f}$ любой новый вектор и рассмотрим полученное множество как множество выполняющих векторов некоторой функции $f^{\prime}$. Тогда $f^{\prime} \in F_{n_{0}, m+1}$ и $E_{f} \subset E_{f^{\prime}}$. Если у функции $f^{\prime}$ существует имплицента $g$ от $k$ переменных, то, по определению, $E_{f^{\prime}} \subset E_{g}$. Так как, по построению $f^{\prime}$, справедливо включение $E_{f} \subset E_{f^{\prime}}$, верно, что $E_{f} \subset E_{g}$, то есть $g$ - имплицента функции $f$, что приводит к противоречию. Следовательно, у функции $f^{\prime}$ также нет имплицент от $k$ переменных, то есть $N_{n_{0}, m+1, k} \neq \varnothing$, что дает неравенство

$$
n_{\max }^{(k)}(m+1) \geqslant n_{0}=n_{\max }^{(k)}(m) .
$$

Пункт 8 доказан.

Из п. 3 и 8 доказанной леммы следует, что функция $n_{\max }^{(k)}(m)$ является возрастающей по $m$ и убывающей по $k$. Далее, в частности, получен вывод о том, что функция $n_{\max }^{(k)}(m)$ не является строго возрастающей по $m$.

Из определения функции $n_{\max }^{(k)}(m)$ легко получить следующее свойство.

Лемма 2. Если $f \in F_{n, m} u n>n_{\max }^{(k)}(m)$, то у функичии $f$ сущчествует имплицента от $k$ переменных.

Это свойство позволит сформулировать некоторые из выводов леммы 1 следующим образом. Имплиценты длины 1 существуют у всех функций веса 1 ; для любого значения веса, большего 1, найдется функция без имплицент длины 1. Функция веса, меньшего $2^{k}$, $k \geqslant 2$, всегда имеет имплиценту длины $k$. Функция веса $m$, зависящая более чем от $2^{m}$ переменных, всегда имеет имплиценты от любого числа переменных, больше либо равного двум.

В [2] в несколько другой терминологии найдено точное значение функции $n_{\max }^{(2)}(m)$.

Как видно из п. 4 леммы 1, существует верхняя оценка величины $n_{\max }^{(k)}(m)$. В настоящей работе для значений $m$, удовлетворяющих неравенству $m<3 \cdot 2^{k-1}$, получены другие, более точные оценки, и, в ряде случаев, точные значения функции $n_{\max }^{(k)}(m)$. 


\section{2. Представление в виде комбинаторно полных матриц}

Существует представление булевых функций, не имеющих имплицент длины $k$ или меньше, в виде специальных матриц. Приведем описание модели с доказательством некоторых свойств.

Двоичная матрица $B$ размера $m \times n, n \geqslant k$, называется комбинаторно полной порядка $k$, если в любой ее подматрице размера $m \times k$ для любого булевого вектора $\vec{v} \in V_{k}$ найдется строка, равная $\vec{v}$. Иначе говоря, при выборе любых $k$ столбцов в матрице $B$ полученная подматрица должна содержать каждую из $2^{k}$ возможных строк (но необязательно только по одному разу). Обозначим через $n_{\max }^{(k)}(m)$ максимальное число столбцов, при котором существует комбинаторно полная порядка $k$ матрица размера $m \times n_{\max }^{(k)}(m)$. Максимальной комбинаторно полной матрицей порядка $k$ будем называть комбинаторно полную матрицу порядка $k$ размера $m \times n_{\max }^{(k)}(m)$.

Приведем некоторые очевидные свойства комбинаторно полных матриц.

Лемма 3. Пусть $B-$ комбинаторно полная порядка $k$ матрицุа размера $m \times n, n \geqslant k$. Тогда справедливы следующие свойства:

$-m \geqslant 2^{k}$

- если $k>1$, то В является комбинаторно полной порядка $k^{\prime}$ для любого $1 \leqslant k^{\prime}<k$;

- любая подматрийа $B$, содержащая $m$ строк и не менее $k$ столбцов, является комбинаторно полной порядка $k$;

- если матрица $B^{\prime}$ получена из матрицы В инвертированием любого столбияа (то есть инвертированием всех его элементов), то $B^{\prime}$ является комбинаторно полной порядка $k$;

- если матрица $B^{\prime}$ получена из матрищы В некоторой перестановкой ее столбцов, то $B^{\prime}$ является комбинаторно полной порядка $k$;

- если матрица $B^{\prime}$ получена из матрицьы В некоторой перестановкой ее строк, то $B^{\prime}$ является комбинаторно полной порядка $k$;

- если матрица $B^{\prime}$ получена из матрищьы В добавлением любого числа произвольных строк, то $B^{\prime}$ является комбинаторно полной порядка $k$.

Пусть $f-$ булева функция от $n$ переменных веса $m$. Обозначим через $\widetilde{E}_{f}$ матрицу, строки которой являются выполняющими векторами $f$, то есть

$$
\widetilde{E}_{f}=\left(\begin{array}{c}
\left(\alpha_{1}^{(1)}, \ldots, \alpha_{n}^{(1)}\right) \\
\vdots \\
\left(\alpha_{1}^{(m)}, \ldots, \alpha_{n}^{(m)}\right)
\end{array}\right),
$$

где $\left(\alpha_{1}^{(i)}, \ldots, \alpha_{n}^{(i)}\right) \in E_{f}, i=1, \ldots, m$. Отметим, что число строк матрицы $\widetilde{E}_{f}$ равно $\|f\|=m$, а число столбцов равно количеству переменных функции $f$, то есть $n$.

Далее покажем связь между комбинаторно полными матрицами порядка $k$ и функциями, не имеющими имплицент длины $k$.

Предложение 1. Булева функция $f$, отличная от константы, имеет имплиценту длиныл $k$ тогда и только тогда, когда матрица $\widetilde{E}_{f}$ не является комбинаторно полной порядка $k$. 
Доказательство. Напомним (см. [1]), что имплицента называется элементарной, если существует ее запись в виде элементарной дизъюнкции. Сначала отметим, что отсутствие у булевой функции имплицент длины $k$ приводит к отсутствию у нее элементарных имплицент длины $k$. Пусть $g$ - имплицента функции $f\left(x_{1}, \ldots, x_{n}\right)$, и $g$ имеет $k$ существенных переменных $x_{i_{1}}, \ldots, x_{i_{k}}$. Воспользовавшись заменой переменных $x_{i_{j}}=y_{j}, j=1, \ldots, k$, приходим к функции от $k$ переменных $g\left(y_{1}, \ldots, y_{k}\right)$. Представим функцию $g$ в виде совершенной КНФ:

$$
g=\prod_{\left(a_{1}, \ldots, a_{k}\right) \in V_{n} \backslash E_{g}}\left(y_{1}^{a_{1} \oplus 1} \vee \ldots \vee y_{k}^{a_{k} \oplus 1}\right) .
$$

Рассмотрим любую из элементарных дизъюнкций $K$ этой совершенной КНФ; очевидно, она имеет $k$ существенных переменных. Далее, при любых значениях неизвестных

$$
f K=(f g) K=f(g K)=f g=f,
$$

то есть $K$ является имплицентой функции $f$.

Рассмотрим элементарную дизъюнкцию длины $k$ :

$$
g=x_{i_{1}}^{a_{1}} \vee \ldots \vee x_{i_{k}}^{a_{k}} .
$$

Эта дизъюнкция является имплицентой функции $f$ тогда и только тогда, когда

$$
f g=f\left(x_{i_{1}}^{a_{1}} \vee \ldots \vee x_{i_{k}}^{a_{k}}\right)=f .
$$

Последнее равенство эквивалентно тому, что на всех векторах $\left(x_{1}, \ldots, x_{n}\right)$, таких, что $x_{i_{1}}=a_{1} \oplus 1, x_{i_{2}}=a_{2} \oplus 1, \ldots, x_{i_{k}}=a_{k} \oplus 1$ выполняется равенство $f\left(x_{1}, \ldots, x_{n}\right)=0$; иначе говоря, среди выполняющих векторов функции $f$ нет таких, в которых на местах $i_{1}, \ldots, i_{k}$ стояли бы значения $\left(a_{1} \oplus 1, \ldots, a_{k} \oplus 1\right)$ соответственно. По определению, это происходит тогда и только тогда, когда в столбцах $i_{1}, \ldots, i_{k}$ матрицы $\widetilde{E}_{f}$ не содержится строка $\left(a_{1} \oplus 1, \ldots, a_{k} \oplus 1\right)$, то есть матрица $\widetilde{E}_{f}$ не является комбинаторно полной.

Заметим, что любая двоичная матрица $B$ размера $m \times n, m \leqslant 2^{n}$, не содержащая одинаковых строк, задает булеву функцию $f$ от $n$ переменных веса $m$ следующим образом:

$$
\widetilde{E}_{f}=B \text {. }
$$

Следствие 1. При любых натуральных $m, k, k>1, m \geqslant 2^{k}$, величина $n_{\max }^{(k)}(m)$ равна наибольшему из возможных количеству столбцов комбинаторно полной матриць порядка $k$, содержащей $m$ строк.

Доказательство. Рассмотрим класс комбинаторно полных матриц порядка $k$, содержащих $m$ различных строк, записанных в лексикографическом порядке, и не менее $k+1$ столбцов. Обозначим этот класс через $\alpha_{m, k}$. По предложению 1 , любая булева функция $f$ однозначно задает некоторую комбинаторно полную матрицу из этого класса следующим образом: $\widetilde{E}_{f}=B$. Кроме того, любая матрица из $\alpha_{m, k}$, очевидно, задает некоторую булеву функцию $f$, не имеющую имплицент длины $k$. Следовательно, определено взаимно однозначное соответствие между функциями из объединения $\bigcup_{n \geqslant k+1} N_{n, m, k}$ и матрицами из $\alpha_{m, k}$. Иными словами, величина $n_{\max }^{(k)}(m)$ равна наибольшему из возможных количеству столбцов матрицы из класса $\alpha_{m, k}$. Обозначим это наибольшее значение количества столбцов через $\tilde{n}_{\max }^{(k)}(m)$, тогда

$$
n_{\max }^{(k)}(m)=\tilde{n}_{\max }^{(k)}(m)
$$


для любых $m, k$, удовлетворяющих условиям следствия.

Теперь рассмотрим класс всех комбинаторно полных матриц порядка $k$, содержащих $m$ строк. Обозначим его через $A_{m, k}$. Очевидно, $\alpha_{m, k} \subset A_{m, k}$. Покажем, что в $A_{m, k}$ нет матриц, у которых число столбцов больше $\tilde{n}_{\max }^{(k)}(m)$. Предположим противное: пусть существует такая матрица $B_{m \times n} \in A_{m, n} \backslash \alpha_{m, n}$, что $n>\tilde{n}_{\max }^{(k)}(m)$. Так как $B \notin \alpha_{m, n}$, не выполнено хотя бы одно из следующих условий:

- $B$ содержит не более $k$ столбцов, $n \leqslant k$;

- строки $B$ не расположены в лексикографическом порядке;

- некоторые строки $B$ одинаковы.

Пусть $n \leqslant k$. Так как

$$
n_{\max }^{(k)}(m) \geqslant k+1
$$

по п. 7 леммы 1, верно, что

$$
\tilde{n}_{\max }^{(k)}(m) \geqslant k+1
$$

поэтому

$$
\tilde{n}_{\max }^{(k)}(m)>n,
$$

что невозможно.

Пусть строки $B$ не расположены в лексикографическом порядке. Тогда перестановкой строк добьемся выполнения этого условия и получим матрицу $B^{\prime}$ размера $m \times n$, которая по лемме 3 является комбинаторно полной порядка $k$.

Далее, если некоторые строки $B^{\prime}$ одинаковы, то рассмотрим матрицу $B^{\prime \prime}$ размера $m_{0} \times n$, в которой все повторяющиеся строки $B^{\prime}$ взяты только по одному разу, $m_{0} \leqslant m$. Из определения комбинаторно полных матриц нетрудно видеть, что $B^{\prime \prime}$ также является комбинаторно полной порядка $k$, и $m_{0} \geqslant 2^{k}$ по лемме 3 . Следовательно, $B^{\prime \prime} \in \alpha_{m_{0}, k}$, поэтому

$$
n \leqslant \tilde{n}_{\max }^{(k)}\left(m_{0}\right)
$$

Кроме того, по доказанному выше

$$
\tilde{n}_{\max }^{(k)}\left(m_{0}\right)=n_{\max }^{(k)}\left(m_{0}\right)
$$

а по п. 8 леммы 1

$$
n_{\max }^{(k)}\left(m_{0}\right) \leqslant n_{\max }^{(k)}(m),
$$

поэтому

$$
n \leqslant n_{\max }^{(k)}(m),
$$

что невозможно. 


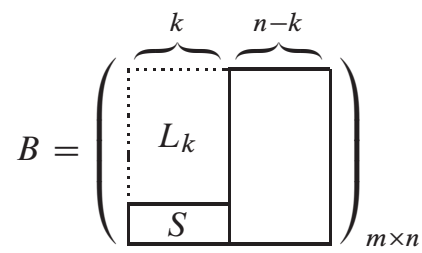

Рис. 1. Приведенная комбинаторно полная матрица порядка $k$

\section{3. Приведенная форма комбинаторно полной матрицы и связанный с ней граф}

Введем следующие определения. Будем обозначать через $L_{k}$ матрицу, составленную из всех строк $V_{k}$ в лексикографическом порядке, то есть

$$
L_{k}=\left(\begin{array}{cccc}
0 & \ldots & 0 & 0 \\
0 & \ldots & 0 & 1 \\
\ldots \ldots & \ldots & \ldots \\
1 & \ldots & 1 & 1
\end{array}\right)
$$

Приведенной комбинаторно полной матрицей порядка $k$ или комбинаторно полной матрицей порядка $k$ в приведенной форме будем называть комбинаторно полную матрицу вида, представленного на рис. 1.

Заметим, что, пользуясь леммой 3 , любую комбинаторно полную порядка $k$ матрицу можно привести к приведенному виду перестановкой строк. При этом приведенная форма для каждой матрицы определена неоднозначно. Далее будем считать, что $n \geqslant k+1$. Определим граф $G(B)$ следующим образом: $G(B)$ - подграф $k$-мерного булева куба $B_{k}$, вершинами $G(B)$ являются строки, содержащиеся в $L_{k}$, но не содержащиеся в $S$. Очевидно, что если $m=2^{k}+s, s<2^{k}$, то множество вершин $G(B)$ непусто. Пусть $W$ - компонента связности графа $G(B)$, вершинами которой являются строки $\vec{l}_{i_{1}}, \vec{l}_{i_{2}}, \ldots, \vec{l}_{i_{t}}$ матрицы $L_{k}, 1 \leqslant i_{1}<i_{2}<\ldots<i_{t} \leqslant 2^{k}$. Для $W$ определим матрицу $M(W)$, которая является подматрицей $B$ и состоит из строк с номерами $i_{1}, i_{2}, \ldots, i_{t}$ и столбцов с номерами $k+1, k+2, \ldots, n$. Покажем, что матрица $M(W)$ будет иметь специальный вид.

Предложение 2. Пусть $m=2^{k}+s, s<2^{k} u W$ - компонента связности графа $G(B)$, не являющаяся изолированной верииной. Тогда матрица $M(W)$ содержит столбиьь не более чем двух видов: $\beta^{\downarrow}$ или $\bar{\beta}^{\downarrow}$.

Доказательство. Положим $B^{\prime}=\left(B_{1}^{\downarrow}, \ldots, B_{k}^{\downarrow}\right)$. Обозначим через $L(W)$ подматрицу $L_{k}$, составленную из строк - вершин $W$. Очевидно, все строки $L(W)$ различны. По условию, $W$ не является изолированной вершиной, поэтому в $W$ есть хотя бы одно ребро, причем смежные вершины в $W$ представляют собой строки, отличающиеся в одной и только в одной координате (по построению $G(B)$ ). Рассмотрим пару таких строк, отличающихся в координате с номером $x \in\{1, \ldots, k\}$ :

$$
\begin{aligned}
& \vec{L}(W)_{i_{1}}=\left(\beta_{1}, \ldots, \beta_{x-1}, \alpha, \beta_{x+1}, \ldots, \beta_{k}\right), \\
& \vec{L}(W)_{i_{2}}=\left(\beta_{1}, \ldots, \beta_{x-1}, \bar{\alpha}, \beta_{x+1}, \ldots, \beta_{k}\right), \quad \alpha, \beta_{q} \in\{0 ; 1\}, \quad q \in\{1, \ldots, k\} \backslash x .
\end{aligned}
$$


Далее, рассмотрим матрицу $B^{\prime \prime}=\left(B_{1}^{\downarrow}, \ldots, B_{x-1}^{\downarrow}, B_{x+1}^{\downarrow}, \ldots, B_{k}^{\downarrow}\right)$; она содержит строку вида $\left(\beta_{1}, \ldots, \beta_{x-1}, \beta_{x+1}, \ldots, \beta_{k}\right)$ по крайней мере дважды: они очевидным образом получаются из строк $\vec{L}(W)_{i_{1}}$ и $\vec{L}(W)_{i_{2}}$ удалением координаты с номером $x$. Пусть это $\vec{B}_{j_{1}}^{\prime \prime}$ и $\vec{B}_{j_{2}}^{\prime \prime}$. Покажем, что $B^{\prime \prime}$ содержит указанную строку не более чем дважды. Действительно, пусть существует $\vec{B}_{j_{3}}^{\prime \prime}=\left(\beta_{1}, \ldots, \beta_{x-1}, \beta_{x+1}, \ldots, \beta_{k}\right)$. Тогда в матрице $B^{\prime}$ строка с номером $j_{3}$ имеет вид $\vec{B}_{j_{3}}^{\prime}=\left(\beta_{1}, \ldots, \beta_{x-1}, \gamma, \beta_{x+1}, \ldots, \beta_{k}\right)$, где $\gamma \in\{0 ; 1\}$. Но тогда $\gamma=\alpha$ или $\gamma=\bar{\alpha}$, поэтому $\vec{B}_{j_{3}}^{\prime}=\vec{L}(W)_{i_{1}}$ или $\vec{B}_{j_{3}}^{\prime}=\vec{L}(W)_{i_{2}}$. Следовательно, $\vec{B}_{j_{3}}^{\prime}$ не может принадлежать матрице $L_{k}$, поскольку все ее строки различны. Кроме того, $\vec{B}_{j_{3}}^{\prime}$ не лежит в $S$, поскольку в этом случае $\vec{L}(W)_{i_{1}}$ или $\vec{L}(W)_{i_{2}}$ не лежали бы в $L(W)$ по построению $G(B)$. Но тогда $\vec{B}_{j_{3}}^{\prime}$ не принадлежит $B^{\prime}$, что невозможно. Таким образом, матрица $B^{\prime \prime}$ содержит строку вида $\left(\beta_{1}, \ldots, \beta_{x-1}, \beta_{x+1}, \ldots, \beta_{k}\right)$ ровно дважды.

Так как матрица $B$ комбинаторно полна, то ее подматрица, составленная из столбцов $\left(B_{1}^{\downarrow}, \ldots, B_{x-1}^{\downarrow}, B_{x+1}^{\downarrow}, \ldots, B_{k}^{\downarrow}, B_{k+1}^{\downarrow}\right)$, содержит обе строки $\left(\beta_{1}, \ldots, \beta_{x-1}, \beta_{x+1}, \ldots, \beta_{k}, 0\right)$ и $\left(\beta_{1}, \ldots, \beta_{x-1}, \beta_{x+1}, \ldots, \beta_{k}, 1\right)$. По доказанному выше, у данной подматрицы существуют только две строки вида $\left(\beta_{1}, \ldots, \beta_{x-1}, \beta_{x+1}, \ldots, \beta_{k}, *\right)$ - это строки с номерами $j_{1}$ и $j_{2}$. Следовательно, $b_{j_{1}, k+1}=\bar{b}_{j_{2}, k+1}$. Итак, если строки $\vec{B}_{j_{1}}^{\prime}$ и $\vec{B}_{j_{2}}^{\prime}$ принадлежат матрице $L(W)$ и отличаются в одной и только в одной координате, то $b_{j_{1}, k+1}=\bar{b}_{j_{2}, k+1}$.

Поскольку $W-$ компонента связности, то для любых двух строк $\vec{L}(W)_{i_{1}}, \vec{L}(W)_{i_{2}} \in$ $L(W)$ существует такая последовательность строк $\vec{L}(W)_{j_{1}}, \ldots, \vec{L}(W)_{j_{p}}$, что $j_{1}=i_{1}$, $j_{p}=i_{2}$, и для любого $r \in\{1, \ldots, p-1\}$ строки $\vec{L}(W)_{j_{r}}$ и $\vec{L}(W)_{j_{r+1}}$ отличаются в одной и только в одной координате. Пусть $L(W)$ состоит из строк $\vec{B}_{j_{1}}^{\prime}, \ldots, \vec{B}_{j_{t}}^{\prime}$. Тогда можно определить весь набор $\left(b_{j_{1}, k+1}, \ldots, b_{j_{t}, k+1}\right)$ однозначно с точностью до выбора $b_{j_{1}, k+1}$. Следовательно, набор $\left(b_{j_{1}, k+1}, \ldots, b_{j_{t}, k+1}\right)$ может быть только двух видов. Докажем, что эти два вида будут отличаться инвертированием всего набора.

Выше было показано, что если $\vec{B}_{j_{1}}^{\prime}$ и $\vec{B}_{j_{2}}^{\prime}$ принадлежат матрице $L(W)$ и отличаются в одной и только в одной координате, то $b_{j_{1}, k+1}=\bar{b}_{j_{2}, k+1}$. Векторы $\vec{B}_{j_{1}}^{\prime}$ и $\vec{B}_{j_{2}}^{\prime}$, очевидно, имеют разную четность. Поэтому в наборе $\left(b_{j_{1}, k+1}, \ldots, b_{j_{t}, k+1}\right)$ одинаковые элементы соответствуют строкам $L(W)$ одинаковой четности. Следовательно, возможны только два случая. В первом случае в наборе $\left(b_{j_{1}, k+1}, \ldots, b_{j_{t}, k+1}\right)$ на местах, соответствующих четным строкам $L(W)$, стоят единицы, а на местах, соответствующих нечетным строкам $L(W)$, стоят нули. Во втором случае в наборе $\left(b_{j_{1}, k+1}, \ldots, b_{j_{t}, k+1}\right)$ на местах, соответствующих четным строкам $L(W)$, стоят нули, а на местах, соответствующих нечетным строкам $L(W)$, стоят единицы. Очевидно, второй случай отличается от первого инвертированием всего набора $\left(b_{j_{1}, k+1}, \ldots, b_{j_{t}, k+1}\right)$. Аналогичные рассуждения можно провести для любого столбца из $\left(B_{k+1}^{\downarrow}, \ldots, B_{n}^{\downarrow}\right)$. Осталось заметить, что матрица $M(W)$ представляет собой подматрицу $B$, составленную из строк с номерами $j_{1}, \ldots, j_{t}$ и столбцов с номерами $k+1, \ldots, n$.

Предложение 3. В условиях предложения 2 матрица $M(W)$ имеет строки только двух видов: $\vec{\gamma}$ или $\overrightarrow{\bar{\gamma}}$.

Доказательство. Рассмотрим матрицу $C=\left(C_{1}^{\downarrow}, \ldots, C_{n-k}^{\downarrow}\right)$, имеющую столбцы только 
двух видов: $\beta^{\downarrow}=\left(b_{1}, \ldots, b_{n-k}\right)^{T}$ или $\bar{\beta}^{\downarrow}=\left(\bar{b}_{1}, \ldots, \bar{b}_{n-k}\right)^{T}$. Положим

$$
\begin{aligned}
(\vec{\beta})^{x} & = \begin{cases}\left(b_{1}, \ldots, b_{k}\right), & x=1, \\
\left(\bar{b}_{1}, \ldots, \bar{b}_{k}\right), & x=0 ;\end{cases} \\
\left(\beta^{\downarrow}\right)^{x} & =\left((\vec{\beta})^{x}\right)^{T} .
\end{aligned}
$$

Тогда

$$
C=\left(\left(\beta^{\downarrow}\right)^{x_{1}}, \ldots,\left(\beta^{\downarrow}\right)^{x_{n-k}}\right), \quad x_{j} \in\{0 ; 1\}, \quad j=1, \ldots, n-k .
$$

Любая строка матрицы $C$ выглядит следующим образом:

$$
\vec{C}_{i}=\left(b_{i}^{x_{1}}, b_{i}^{x_{2}}, \ldots, b_{i}^{x_{n-k}}\right)=\left(x_{1}^{b_{i}}, x_{2}^{b_{i}}, \ldots, x_{n-k}^{b_{i}}\right)=(\vec{x})^{b_{i}} .
$$

Следовательно, строки матрицы $C$ могут быть только двух видов: $\vec{x}$ или $\overrightarrow{\bar{x}}$.

Далее, воспользуемся предложением 3, чтобы сформулировать и доказать теорему, описывающую свойства графа $G(B)$.

Теорема 1. Пусть $B_{0}-$ комбинаторно полная порядка $k$ матрица размера $m \times n$, где $m=2^{k}+s, s<2^{k}, n \geqslant k+1 ;$ пусть $B-$ приведенная форма матрицы $B_{0}$. Если граф $G(B)$ содержит $t_{1}$ изолированных вершин и $t_{2}$ компонент связности порядка не менее 2 , mo:

$$
\begin{array}{ll}
2 s \geqslant 2^{k}-t_{1}-2 t_{2} & \text { если } n \geqslant 2 k, \\
2 s \geqslant 2^{k}-t_{1}-2^{2 k-n+1} t_{2} & \text { если } n<2 k .
\end{array}
$$

Доказательство. Пусть $W_{i}, i=1, \ldots, t_{1}+t_{2},-$ все компоненты связности графа $G(B)$. Каждая компонента связности - подматрица $L_{k}$, причем подматрицы $W_{i}, i=1, \ldots, t_{1}$, содержат одну строку, остальные содержат не менее двух строк. Тогда матрицы $M\left(W_{i}\right)$, $i=1, \ldots, t_{1}$, содержат по одной строке, и по предложению 3 каждая матрица $M\left(W_{i}\right)$, $i=t_{1}+1, \ldots, t_{2}$, содержит не более двух различных строк. Следовательно, всего в матрицах $M\left(W_{i}\right), i=1, \ldots, t_{1}+t_{2}$, содержится не более $t_{1}+2 t_{2}$ различных строк. Если матрица $S$ (см. рис. 1) содержит $s^{\prime}$ различных строк, то граф $G(B)$ состоит из $2^{k}-s^{\prime}$ вершин, поэтому всего в матрицах $M\left(W_{i}\right), i=1, \ldots, t_{1}+t_{2}$, содержится $2^{k}-s^{\prime}$ строк.

Если $n \geqslant 2 k$, то положим $B^{\prime}=\left(B_{k+1}^{\downarrow}, B_{k+2}^{\downarrow}, \ldots, B_{2 k}^{\downarrow}\right)$ и обозначим через $m\left(W_{i}\right)$ подматрицу $B^{\prime}$, составленную из всех общих элементов $B^{\prime}$ и $M\left(W_{i}\right), i=1, \ldots, t_{1}+t_{2}$. Из сказанного выше следует, что матрицы $m\left(W_{i}\right), i=1, \ldots, t_{1}+t_{2}$, содержат в общем счете $2^{k}-s^{\prime}$ строк, из которых не более $t_{1}+2 t_{2}$ различных. Для комбинаторной полноты матрицы $B$ необходимо наличие $2^{k}$ различных строк в $B^{\prime}$. При этом $B^{\prime}$ содержит только $s^{\prime}+s$ строк, не принадлежащих матрицам $m\left(W_{i}\right), i=1, \ldots, t_{1}+t_{2}$. Следовательно, $s^{\prime}+s \geqslant 2^{k}-\left(t_{1}+2 t_{2}\right)$. Осталось заметить, что $s \geqslant s^{\prime}$, поэтому $2 s \geqslant 2^{k}-t_{1}-2 t_{2}$.

Если $n<2 k$, то положим $B^{\prime}=\left(B_{k+1}^{\downarrow}, B_{k+2}^{\downarrow}, \ldots, B_{n}^{\downarrow}\right)$ и аналогично определим матрицы $m\left(W_{i}\right), i=1, \ldots, t_{1}+t_{2}$. Пусть $B^{\prime \prime}=\left(B_{n-k+1}^{\downarrow}, \ldots, B_{k}^{\downarrow}, B_{k+1}^{\downarrow}, \ldots, B_{n}^{\downarrow}\right)$, обозначим ее строки $\vec{\beta}^{(i)}=\left(\beta_{1}^{(i)}, \ldots, \beta_{2 k-n}^{(i)}, \beta_{2 k-n+1}^{(i)}, \ldots, \beta_{k}^{(i)}\right), i=1, \ldots, 2^{k}+s$. Как и в случае $n \geqslant 2 k$, матрицы $m\left(W_{i}\right), i=1, \ldots, t_{1}+t_{2}$, содержат не более $t_{1}+2 t_{2}$ различных строк. Поэтому строк $\vec{\beta}^{(i)}$, для которых $\left(\beta_{2 k-n+1}^{(i)}, \ldots, \beta_{k}^{(i)}\right)$ принадлежит некоторой матрице $m\left(W_{i}\right)$, $i=1, \ldots, t_{1}+t_{2}$, может быть не более чем $t_{1}+2^{2 k-n} \cdot 2 t_{2}$ вариантов. Остальных строк $\vec{\beta}^{(i)}$ в $B^{\prime}$ содержится $s^{\prime}+s$, поэтому для комбинаторной полноты матрицы $B$ необходимо выполнение условия $s^{\prime}+s \geqslant 2^{k}-\left(t_{1}+2^{2 k-n} \cdot 2 t_{2}\right)$, поэтому $2 s \geqslant 2^{k}-t_{1}-2^{2 k-n+1} t_{2}$. 


\section{4. Оценки величины $n_{\max }^{(k)}(m)$ при небольших значениях $m$}

Начнем с минимального значения $m=2^{k}$.

Предложение 4. Для любого натурального $k>1$ справедливо равенство

$$
n_{\max }^{(k)}\left(2^{k}\right)=k+1 .
$$

Доказательство. Пусть

$$
n_{\max }^{(k)}\left(2^{k}\right) \geqslant k+2 .
$$

Тогда существует комбинаторно полная порядка $k$ матрица, содержащая $2^{k}$ строк и $k+2$ столбцов. Перестановкой строк приведем матрицу $B$ к каноническому виду. Очевидно, граф $G(B)$ совпадает с $B_{k}$ и имеет единственную компоненту связности, содержащую все строки из $V_{k}$. Поэтому определенная выше матрица $M\left(B_{k}\right)$ будет иметь вид $M\left(B_{k}\right)=\left(B_{k+1}^{\downarrow}, B_{k+2}^{\downarrow}\right)$. По предложению 3 , матрица $M\left(B_{k}\right)$ содержит строки только двух видов. Так как матрица $B$ является комбинаторно полной порядка $k \geqslant 2$, то по лемме 3 она должна быть комбинаторно полной порядка 2. Однако подматрица $\left(B_{k+1}^{\downarrow}, B_{k+2}^{\downarrow}\right)$ содержит строки только двух видов, поэтому $B$ не является комбинаторно полной порядка 2, приходим к противоречию. Следовательно,

$$
n_{\max }^{(k)}\left(2^{k}\right) \leqslant k+1 \text {. }
$$

Но по п. 7 леммы 1 верно обратное неравенство

$$
n_{\max }^{(k)}\left(2^{k}\right) \geqslant k+1
$$

таким образом, верно равенство

$$
n_{\max }^{(k)}\left(2^{k}\right)=k+1
$$

Теорема 1 позволяет получить ограничения на величину $n_{\max }^{(k)}(m)$ для небольших значений $m$.

Предложение 5. Пусть $k \geqslant 3, m=2^{k}+s, s<k$. Тогда справедлива оценка

$$
n_{\max }^{(k)}(m) \leqslant 2 k-\log _{2}\left(2^{k-1}-(k-1)\right) .
$$

Доказательство. Пусть матрица $B$ размера $m \times n$ является комбинаторно полной порядка $k$ в приведенной форме. Булев куб $B_{k}$ является $k$-связным графом. Так как по условию $s<k$, то граф $G(B)$ получен из $B_{k}$ удалением не более чем $k-1$ вершин. Следовательно, $G(B)$ содержит только одну компоненту связности $W$ и не содержит изолированных вершин. По теореме 1 , если $n \geqslant 2 k$, то $2 s \geqslant 2^{k}-2$, а так как $s<k$, верно, что $k>2^{k-1}-1$. При $k \geqslant 3$ последнее неравенство не выполняется, поэтому $n<2 k$. Следовательно, по теореме 1

$$
2 s \geqslant 2^{k}-2^{2 k-n+1},
$$

поэтому

$$
(k-1) \geqslant 2^{k-1}-2^{2 k-n}, \quad n \leqslant 2 k-\log _{2}\left(2^{k-1}-(k-1)\right) .
$$


При $k \geqslant 4$ можно уточнить оценку, полученную в предложении 5 . Случай $k=3$ рассмотрим отдельно в следующем примере.

Пример 2. Пусть $k=3, m=2^{k}+s, s<k$. Воспользовавшись результатом предложения 5, получаем оценку

$$
n_{\max }^{(3)}(10) \leqslant 5 \text {. }
$$

Существует матрица, для которой достигается эта оценка. Действительно, рассмотрим матрицу

$$
B=\left(\begin{array}{llllllllll}
1 & 1 & 1 & 0 & 1 & 0 & 0 & 0 & 0 & 1 \\
0 & 1 & 1 & 0 & 1 & 0 & 0 & 1 & 1 & 0 \\
0 & 1 & 0 & 1 & 0 & 1 & 0 & 1 & 0 & 1 \\
0 & 0 & 1 & 1 & 0 & 0 & 1 & 1 & 0 & 1 \\
0 & 0 & 0 & 0 & 1 & 1 & 1 & 1 & 0 & 1
\end{array}\right)_{5 \times 10}
$$

Непосредственной проверкой можно убедиться, что матрица $B^{T}$ является комбинаторно полной порядка 3. Таким образом,

$$
n_{\max }^{(3)}(10)=5 \text {. }
$$

Известно также, что

$$
n_{\max }^{(3)}(8)=4
$$

(по предложению 4). Поскольку функция $n_{\max }^{(k)}(m)$ не убывает по $m$, то $n_{\max }^{(3)}(9) \in\{4 ; 5\}$. Предположим, что $n_{\max }^{(3)}(9)=5$. При $m=9$ граф $G(B)$ содержит одну компоненту связности порядка $2^{k}-1$ и не имеет изолированных вершин. Следовательно, по теореме 1(b) имеем $2 \geqslant 8-4$, что невозможно. Следовательно,

$$
n_{\max }^{(3)}(9)=4 \text {. }
$$

Далее, рассмотрим случай комбинаторно полных матриц порядка $k \geqslant 4$.

Теорема 2. Пусть $k \geqslant 4, m=2^{k}+s, s<k$. Тогда справедливо тождество:

$$
n_{\max }^{(k)}(m)=k+1 \text {. }
$$

Доказательство. Рассмотрим функцию

$$
f(x)=x-1-\log _{2} x .
$$

Исследуем ее на монотонность:

$$
f^{\prime}(x)=1-\frac{1}{x \ln 2}
$$

При $x \geqslant 2 f^{\prime}(x)>0$, поэтому $f(x)$ строго возрастает на промежутке $[2 ;+\infty)$. Так как $f(2)=0$, при $x \geqslant 3 f(x)>0$, то есть $x-1-\log _{2} x>0$. Следовательно, $2^{x-1}>x$. Поскольку $k \geqslant 4$, в последнем неравенстве произведем замену $x=k-1$ и получим $2^{k-2}>k-1$, что эквивалентно неравенству $\left(2^{k-1}-2^{k-2}\right)>k-1$. Тогда

$$
\log _{2}\left(2^{k-1}-(k-1)\right)>k-2 \text {. }
$$


А так как по предложению 5

$$
n_{\max }^{(k)}(m) \leqslant 2 k-\log _{2}\left(2^{k-1}-(k-1)\right),
$$

верно неравенство $n_{\max }^{(k)}(m)<k+2$, поэтому $n_{\max }^{(k)}(m) \leqslant k+1$. По п. 7 леммы 1 , верно неравенство $n_{\max }^{(k)}(m) \geqslant k+1$ и в итоге получаем, что

$$
n_{\max }^{(k)}(m)=k+1 \text {. }
$$

Следствие 2. При $k \geqslant 3$ функц̧ия $n_{\max }^{(k)}(m)$ не является строго возрастающей.

Доказательство. При $k=3$, согласно примеру 2 ,

$$
n_{\max }^{(3)}(9)=4
$$

С другой стороны, по предложению 4,

$$
n_{\max }^{(3)}(8)=4 \text {, }
$$

поэтому при $k=3$ функция $n_{\max }^{(k)}(m)$ возрастает нестрого. Отметим, что это не единственное исключение - пусть $k \geqslant 4$, тогда по теореме 2

$$
n_{\max }^{(k)}\left(2^{k}+k-1\right)=n_{\max }^{(k)}\left(2^{k}+k-2\right)=\ldots=n_{\max }^{(k)}\left(2^{k}\right)=k+1 .
$$

Предложение 3 можно использовать для получения оценки числа столбцов комбинаторно полной матрицы $B$, для которой граф $G(B)$ содержит достаточно большую компоненту связности, не являющуюся изолированной вершиной или ребром. Чем больше порядок компоненты связности, тем лучшую оценку числа столбцов можно получить, используя следующее утверждение.

Предложение 6. Пусть $B_{0}-$ комбинаторно полная порядка $k$ матрица размера $m \times n$, где $m=2^{k}+s, s<2^{k}, n \geqslant 2 k$; и пусть $B-$ приведенная форма матрицьь $B_{0}$. Если граф $G(B)$ содержит компоненту связности порядка $l \geqslant 3$, то справедлива оценка

$$
n \leqslant n_{\max }^{(k)}(m-[(l+1) / 2]+1)+\min \{k ; l-1\} .
$$

Доказательство. Пусть $W$ - компонента связности графа $G(B)$ порядка $l$. Тогда по предложению 3 матрица $M(W)$ содержит строки только двух видов, поэтому в $M(W)$ существует, по крайней мере, $[(l+1) / 2]$ одинаковых строк. Если $l \geqslant k$, то рассмотрим матрицу $B^{\prime}=\left(B_{k+1}^{\downarrow}, B_{k+2}^{\downarrow}, \ldots, B_{2 k}^{\downarrow}\right)$, которая содержит $M(W)$ как подматрицу, и поэтому также имеет, по крайней мере, $[(l+1) / 2]$ одинаковых строк. Покажем, что $B^{\prime}$ содержит не более $n_{\max }^{(k)}(m-[(l+1) / 2]+1)$ столбцов. Действительно, предположим, что $B^{\prime}$ содержит хотя бы $n_{\max }^{(k)}(m-[(l+1) / 2]+1)+1$ столбцов. Тогда рассмотрим $B^{\prime \prime}-$ подматрицу $B^{\prime}$, в которой из $[(l+1) / 2]$ одинаковых строк матрицы $B^{\prime}$ взята только одна, то есть $B^{\prime \prime}$ имеет размер $(m-[(l+1) / 2]+1) \times\left(n_{\max }^{(k)}(m-[(l+1) / 2]+1)+1\right)$. Матрица $B^{\prime}$ является комбинаторно полной порядка $k$, поэтому $B^{\prime \prime}$ также является комбинаторно полной порядка $k$, поскольку в ней не изменилось число различных строк. Тогда существует комбинаторно полная порядка $k$ матрица размером $(m-[(l+1) / 2]+1) \times\left(n_{\max }^{(k)}(m-[(l+1) / 2]+1)+1\right)$, что противоречит определению $n_{\max }^{(k)}(m)$. Итак, $B^{\prime}$ содержит не более чем $n_{\max }^{(k)}(m-[(l+1) / 2]+1)$ столбцов, поэтому $B$ содержит не более $n_{\max }^{(k)}(m-[(l+1) / 2]+1)+k$ столбцов. 
Пусть теперь $l<k$. Тогда в $W$ не существует строк, отличающихся более чем в $l-1$ координатах, поэтому все строки $W$ содержат $k-l+1$ координат, значение которых постоянно. Пусть это координаты с номерами $i_{1}, i_{2}, \ldots, i_{k-l+1}$. Тогда положим $B^{\prime}=\left(B_{i_{1}}^{\downarrow}, B_{i_{2}}^{\downarrow}, \ldots, B_{i_{k-l+1}}^{\downarrow}, B_{k+1}^{\downarrow}, B_{k+2}^{\downarrow}, \ldots, B_{2 k}^{\downarrow}\right)$. Как и в случае $l \geqslant k, B^{\prime}$ содержит не более $n_{\max }^{(k)}(m-[(l+1) / 2]+1)$ столбцов, поэтому $B$ содержит не более $n_{\max }^{(k)}(m-[(l+1) / 2]+1)+l-1$ столбцов.

Доказанная в утверждении 6 оценка формально верна и для случаев $l=1$ или $l=2$. Однако полученные неравенства не несут содержательной нагрузки. В частности, подставив $l=1$, получим $n \leqslant n_{\max }^{(k)}(m)$, а при $l=2$ имеем соотношение $n \leqslant n_{\max }^{(k)}(m)+1$. Покажем, при каком значении $m$ числа строк матрицы $B$ граф $G(B)$ точно содержит компоненту связности порядка $l \geqslant 3$.

Предложение 7. Пусть $B_{0}-$ комбинаторно полная порядка $k$ матрица, содержащая $m$ строк, где $m=2^{k}+s, s<2^{k} ;$ и пусть $B-$ приведенная форма матрицьь $B_{0}$. Если $s<2^{k-1}$, то $G(B)$ содержит компоненту связности порядка $l \geqslant 3$.

Доказательство. Если $s<2^{k}$, то граф $G(B)$ получен из $k$-мерного булева куба $B_{k}$ удалением менее $2^{k-1}$ вершин. Обозначим через $S$ множество удаленных из $B_{k}$ вершин, $|S|<2^{k-1}$. Индукцией по $k \geqslant 2$ докажем следующий факт, из которого будет следовать искомое утверждение: если граф $G$ получен из $B_{k}$ удалением менее $2^{k-1}$ вершин, то он содержит компоненту связности порядка $l \geqslant 3$.

Пусть $k=2$. Следовательно, граф $G$ получен из $B_{2}$ удалением одной вершины или совпадает с $B_{2}$, поэтому $G$ содержит компоненту связности порядка $l \geqslant 3$.

Пусть теперь $k>2$, и пусть утверждение верно для любого $k^{\prime}, 2 \leqslant k^{\prime}<k$. Докажем его справедливость для $k^{\prime}=k$. Булев куб можно представить в виде объединения двух его граней, то есть подграфов, образованных фиксацией любой координаты некоторым значением. Пусть, например, $B_{k}=\Gamma_{1}^{(0)} \cup \Gamma_{1}^{(1)}$, где $\Gamma_{1}^{(j)}-$ все вершины $B_{k}$, у которых первая координата равна $j, j \in\{0,1\}$. Тогда $\Gamma_{1}^{(0)} \cap \Gamma_{1}^{(1)}=\varnothing$. Далее,

$$
S=S \cap B_{k}=\left(S \cap \Gamma_{1}^{(0)}\right) \cup\left(S \cap \Gamma_{1}^{(1)}\right),
$$

причем $\left(S \cap \Gamma_{1}^{(0)}\right) \cap\left(S \cap \Gamma_{1}^{(1)}\right)=\varnothing$. Следовательно,

$$
|S|=\left|S \cap \Gamma_{1}^{(0)}\right|+\left|S \cap \Gamma_{1}^{(1)}\right|<2^{k-1},
$$

как было сказано выше. Для выполнения последнего неравенства необходимо, чтобы для некоторого $j \in\{0,1\}$ выполнялось условие $\left|S \cap \Gamma_{1}^{(j)}\right|<2^{k-2}$. Грань $\Gamma_{1}^{(j)}$ представляет собой $(k-1)$-мерный булев куб $B_{k-1}$ с точностью до обозначения вершин. Положим $S^{\prime}=S \cap \Gamma_{1}^{(j)},\left|S^{\prime}\right|<2^{k-2}$, и воспользуемся предположением индукции для графа $G^{\prime}=$ $\Gamma_{1}^{(j)} \backslash S^{\prime}$. Тогда $G^{\prime}$ содержит компоненту связности порядка $l \geqslant 3$, а так как граф $G^{\prime}$ является подграфом $G$, то $G$ содержит компоненту связности порядка $l \geqslant 3$.

Пользуясь предложениями 6 и 7, можно получить очередную оценку для $n_{\max }^{(k)}(m)$.

Предложение 8. Пусть $m=2^{k}+s, k \leqslant s<2^{k-1}, k \geqslant 4$. Тогда справедлива оценка

$$
n_{\max }^{(k)}(m) \leqslant k(s-k+2)+1 \text {. }
$$


Доказательство. Пусть $B$ - максимальная комбинаторно полная порядка $k$ матрица размера $m \times n$ в приведенной форме, $n=n_{\max }^{(k)}$. Если $n<2 k$, то доказываемая оценка верна. Действительно, так как по условию $s \geqslant k$, верно, что

$$
2 k<k(s-k+2)+1 \text {. }
$$

Далее считаем, что $n \geqslant 2 k$.

Согласно предложению 7 , граф $G(B)$ содержит компоненту связности порядка $l \geqslant 3$. Следовательно, по предложению 6 справедлива оценка

$$
n \leqslant n_{\max }^{(k)}(m-[(l+1) / 2]+1)+\min \{k ; l-1\} \leqslant n_{\max }^{(k)}(m-1)+k .
$$

Далее, нетрудно убедиться, что если комбинаторно полная порядка $k$ матрица $B^{\prime}$ в приведенной форме имеет $m-i$ строк, $i=0, \ldots, s$, то граф $G\left(B^{\prime}\right)$ также содержит компоненту связности порядка $l \geqslant 3$, поэтому для $B^{\prime}$ также можно воспользоваться оценкой из предложения 6. Следовательно, справедлива цепочка неравенств

$$
\begin{aligned}
n & \leqslant n_{\max }^{(k)}(m-1)+k \leqslant n_{\max }^{(k)}(m-2)+2 k \\
& \leqslant \ldots \leqslant n_{\max }^{(k)}\left(2^{k}+k-1\right)+\left(m-2^{k}+k+1\right) k .
\end{aligned}
$$

Но по теореме 2

$$
n_{\max }^{(k)}\left(2^{k}+k-1\right)=k+1
$$

Подставив

$$
n=n_{\max }^{(k)}(m), \quad m=2^{k}+s,
$$

получим

$$
n_{\max }^{(k)}(m) \leqslant k(s-k+2)+1 .
$$

Согласно предложению 7, если матрица $B$, являясь комбинаторно полной порядка $k$, содержит менее $3 \cdot 2^{k-1}$ строк, то граф $G(B)$ будет содержать компоненту связности порядка $l \geqslant 3$, что позволяет воспользоваться предложением 6 и получить ограничение на число столбцов матрицы $B$. Однако, если матрица содержит хотя бы $3 \cdot 2^{k-1}$ строк, то предложением 6 воспользоваться не удастся, поскольку справедливо следующее утверждение.

Предложение 9. Пусть $m=3 \cdot 2^{k-1}$. Тогда существует такая комбинаторно полная порядка $k$ матрица $B$, содержащая $m$ строк, что граф $G(B)$ не содержит компонент связности порядка $l>1$.

Доказательство. Рассмотрим комбинаторно полную порядка $k$ матрицу $B$, содержащую $m$ строк, следующего вида. Пусть $B$ дана в некоторой приведенной форме, и пусть матрица $S$ (см. рис. 1) размера $2^{k-1} \times k$ не содержит одинаковых строк, причем все ее строки имеют одинаковую четность. Булев куб $B_{k}$ является двудольным графом, причем каждая его вершинная доля - это совокупность векторов одинаковой четности. Следовательно, при таком выборе $S$ множество вершин графа $G(B)$ представляет собой вершинную долю $B_{k}$, но вершинная доля не может содержать ребер, поэтому $G(B)$ в этом случае будет состоять только из изолированных вершин. 


\section{Список литературы}

1. Самофалов К. Г., Романкевич А. М., Валуйский В. Н., Каневский Ю. С., Пиневич М. М., Прикладная теория цифровых автоматов. Вища школа, Киев, 1987.

2. Ролдугин П. В., Тарасов А. В., О булевых функциях без верхних биюнктивных аналогов. Математические вопросы криптографии (2013) 4, №1, 123-140.

3. Cusick T. W., Stănică P., Cryptographic Boolean functions and applications. Elsevier, Amsterdam, 2009.

4. Яблонский С. В., Введение в дискретную математику. Высшая школа, Москва, 2001.

Статья поступила 15.06.2012. 